\title{
$\underbrace{\pi}_{\text {A Division of the Swiss Chemical Society }}$
}

\section{Ultra-Sensitive Measurement of Ocean pH}

\section{Pitchnaree Kraikaew and Eric Bakker}

${ }^{*}$ Correspondence: Prof. Dr. E. Bakker, E-mail: Eric.Bakker@unige.ch Department of Inorganic and Analytical Chemistry, University of Geneva, 30, quai Ernest-Ansermet, $\mathrm{CH}-1211$ Geneva

Keywords: Capacitive readout $\cdot$ Capacitor $\cdot$ Ion-selective electrode $\cdot \mathrm{pH}$ measurement $\cdot$ Seawater

When S. Sørensen started to quantify solution acidity in 1909 by introducing the now ubiquitous concept of $\mathrm{pH}$, he did so on the basis of a potentiometric measurement cell. From the Nernst equation this gives a change of the electromotive force that depends on the logarithmic hydrogen ion activity. Because this earliest link between $\mathrm{pH}$ and resulting potential has existed from the start, the practical $\mathrm{pH}$ scale is still based on the output of a properly calibrated potentiometric $\mathrm{pH}$ probe. Today, glass electrodes measured against a standardized reference electrode containing a $3 \mathrm{M} \mathrm{KCl}$ electrolyte in contact with the sample through a liquid junction are most often used, but other electrode materials are also established.

Unfortunately, potentiometric probes give reproducibilities on the order of $\mathrm{mpH}$ units and are therefore not adequate for some applications where exquisite sensitivities are required. One such example is the reliable monitoring of ocean acidification, an anthropogenic problem of potentially enormous significance that requires ultra-high resolution data. In the past 30 years, ocean $\mathrm{pH}$ has decreased by 0.035 units, about $1.2 \mathrm{mpH}$ per year or just 97 $\mu \mathrm{pH}$ per month. This is a difficult measurement science problem.

We have recently reported that $\mathrm{pH}$ measurements from potentiometric probes can be made dramatically more sensitive by operating in a capacitive detection mode. ${ }^{[1,2]}$ It requires a cell potential that is held constant while the sample $\mathrm{pH}$ is compared with that of a reference solution. The unique feature relative to earlier work is the use of an electronic capacitive component placed in series with the $\mathrm{pH}$ probe (labeled as $\mathrm{C}$ in the Figure). Any small $\mathrm{pH}$ deviation between the reference and sample solution still results in the same potential change, but since the cell potential is held constant it must be compensated by an opposite voltage change over the capacitive element. This gives rise to a current spike that is more easily identifiable than the underlying potential change. The current is background-subtracted and integrated to give the corresponding charge, which serves as signal because it is directly proportional to the change of $\mathrm{pH}$. In our initial work with membrane electrodes containing an aqueous inner solution, a reproducibility (standard deviation) of $28 \mu \mathrm{pH}$ in buffer and 67 $\mu \mathrm{pH}$ in stabilized seawater samples was achieved, which is orders of magnitude better than with direct potentiometry. ${ }^{[1]}$

More recently, the concept has been extended to all solid-state membrane electrodes that are pressure-insensitive and therefore more practical for environmental use. ${ }^{[2]}$ The inner ion-to-electron transducing material is already characterized by a capacitance. It is, however, non-ideal and gives rise to sluggish current transients that are very difficult to quantify if no additional external capacitor is added. Kirchhoff's law is useful to choose the appropriate value for the external capacitor as it needs to be chosen to dominate the capacitance of the entire cell. We now aim to make this principle applicable for the electrochemical in situ monitoring of ocean $\mathrm{pH}$ with unsurpassed precision. A capacitive readout method is orders of magnitude more sensitive than direct potentiometry.

Received: November 4, 2020

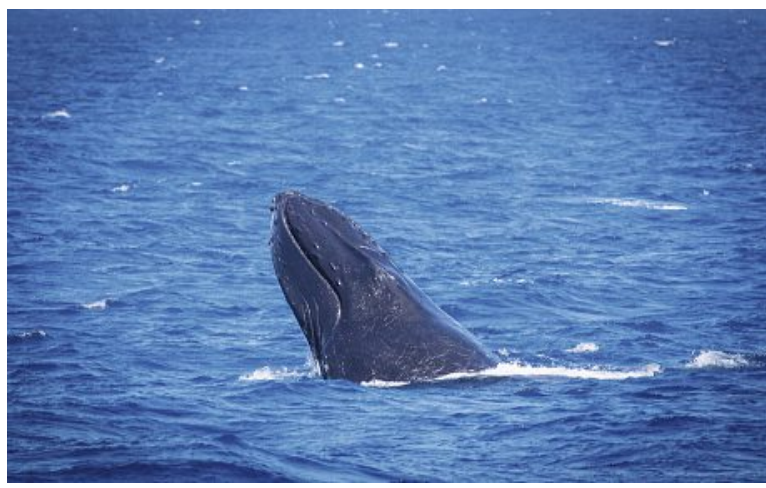

Humpback whale off Sydney Harbour. ( Eric Bakker, 2020.
POTENTIOMETRY
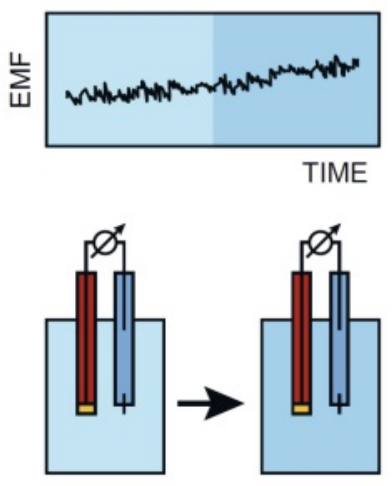

REFERENCE
SAMPLE
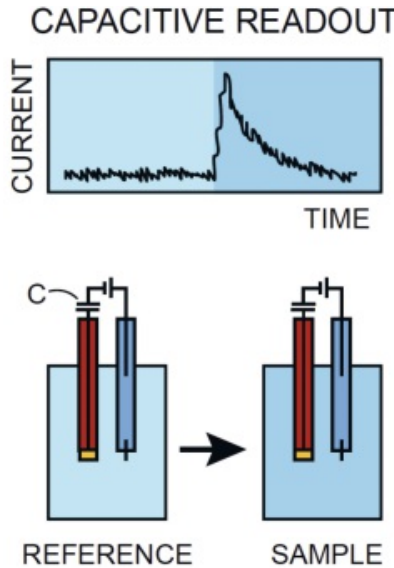

Principles of potentiometry and capacitive readout. Left: With established potentiometric $\mathrm{pH}$ probes, a small $\mathrm{pH}$ change from a reference to a sample solution (bottom) results in a potential change that is often difficult to distinguish from baseline drift (top). Right: With the new methodology the cell potential is held constant and the potential change at the $\mathrm{pH}$ probe results in a charging of the capacitive element $\mathrm{C}$ placed in series (bottom). This results in a current spike that is more easily identified and isolated (top). The final sensor signal is the charge from the integrated current, which is proportional to the capacitance value and the $\mathrm{pH}$ change.

\section{References}

[1] P. Kraikaew, S. Jeanneret, Y. Soda, T. Cherubini, E. Bakker, ACS Sensors 2020, 5, 650 .

[2] P. Kraikaew, S. K. Sailapu, E. Bakker, Anal. Chem. 2020, 92, 14174. 